
\title{
25 Research Soure \\ Predictive Value of Matrix Metalloprotease 9 On Surgical Outcomes After Pericardiectomy
}

\section{Likui Fang}

Affiliated Hangzhou Chest Hospital, Zhejiang University School of Medicine

\section{Wenfeng Yu}

Affiliated Hangzhou Chest Hospital, Zhejiang University School of Medicine

\section{Guocan Yu}

Affiliated Hangzhou Chest Hospital, Zhejiang University School of Medicine

\section{Bo Ye}

Affiliated Hangzhou Chest Hospital, Zhejiang University School of Medicine

Gang Chen ( $D$ cgang06@sina.com )

Affiliated Hangzhou Chest Hospital, Zhejiang University School of Medicine

\section{Research Article}

Keywords: MMP9, constrictive pericarditis, postoperative outcomes

Posted Date: July 26th, 2021

DOl: https://doi.org/10.21203/rs.3.rs-659348/v1

License: (c) (i) This work is licensed under a Creative Commons Attribution 4.0 International License.

Read Full License

Version of Record: A version of this preprint was published at Journal of Cardiothoracic Surgery on March 23rd, 2022. See the published version at https://doi.org/10.1186/s13019-022-01796-9. 


\section{Abstract \\ Background}

The effects of matrix metalloproteases (MMPs) and tissue inhibitors of metalloproteinases (TIMPs) expressions on the patients with constrictive pericarditis undergoing pericardiectomy remain unclear. This study explored the associations of MMPs and TIMPs expressions with postoperative outcomes in these patients.

\section{Methods}

Pericardial specimens were obtained during pericardiectomy from the patients with constrictive pericarditis. The levels of MMP1, MMP2, MMP9 and TIMP1 in pericardium were analyzed by quantitative real-time polymerase chain reaction. The enrolled patients were divided into two groups according to the optimal cutoff value of gene expression predicting postoperative complications. Postoperative outcomes were compared between the two groups. Binary logistic regression analysis was performed to determine the degree of contribution of gene expression on postoperative outcomes.

\section{Results}

A total of 22 patients and their pericardial specimens were included. The level of MMP9 was significantly associated with postoperative complications and the optimal cutoff value predicting postoperative complications was 3.67. The patients with low level of MMP9 $(<3.67)$ had lower incidence of postoperative complications $(P=0.002)$, shorter postoperative intensive care unit $(P=0.040)$ and hospital stay $(P=0.043)$ in comparison to those with high level of MMP9 $(\geq 3.67)$. Binary logistic regression analysis showed that high level of MMP9 increased risk of postoperative complications (OR, 27.096, $95 \% \mathrm{Cl} 1.166-629.886, \mathrm{P}=0.040)$.

\section{Conclusions}

High level of MMP9 in the pericardium was associated with poor postoperative outcomes and was the independent risk factor of postoperative complications. The level of MMP9 could be used as a potential marker for prediction of surgical outcomes.

\section{Background}

Constrictive pericarditis is a rare disease caused by pericardial inflammation, fibrosis and inelasticity[1]. The etiology of constrictive pericarditis is various in different areas. Most cases in Europe and North America are idiopathic or related to prior cardiac surgery or chest irradiation, while tuberculosis is the most common cause in developing counties[2,3]. Constrictive pericarditis is chronic and progressive in 
most cases, and leads to diastolic heart failure with poor quality of life and prognosis[4]. The treatment approaches of constrictive pericarditis are limited. Palliative treatment such as diuretic therapy only reduces symptoms temporarily in chronic cases, and surgical pericardiectomy is the only definitive treatment to relieve the pericardial constriction[5, 6]. However, pericardiectomy is associated with high incidence of postoperative complications and in-hospital mortality[7, 8].

Matrix metalloproteases (MMPs) are a large family of zinc dependent endopeptidases that can degrade almost every component of the extracellular matrix (ECM), and the enzymatic activity of MMPs can be blocked by tissue inhibitors of metalloproteinases (TIMPs) through binding to the active site of MMPs[9]. Abnormal expressions of MMPs and TIMPs are involved in a variety of pathological conditions, including inflammation and fibrosis[10]. In addition, the levels of MMPs have been reported to be associated with surgical outcomes and could be potential biomarkers predicting postoperative complications[11-13]. However, the roles of MMPs and TIMPs in the patients with constrictive pericarditis undergoing pericardiectomy have not been investigated. This study aimed to explore the effects of MMPs and TIMPs expressions on postoperative outcomes in those patients.

\section{Methods}

\section{Study population}

The pericardial specimens were obtained during pericardiectomy from the patients with constrictive pericarditis in our department between January 2018 and November 2019. The tissue specimens were collected from the pericardium over the left ventricle and the size was $0.5 \mathrm{~cm}$ by $0.5 \mathrm{~cm}$. A total of 22 specimens were successfully collected from the patients. We retrospectively reviewed the records of these patients and their characteristics including demographic, preoperative and outcomes data were extracted from the hospital electronic medical records system. The study protocol was approved by the Institutional Review Board of Affiliated Hangzhou Chest Hospital, Zhejiang University School of Medicine (ID of ethics approval: 20160830) and written patient informed consent was obtained.

\section{Interventions and outcomes}

The preoperative diagnosis of constrictive pericarditis mainly depended on the clinical symptoms, echocardiography, chest enhanced computed tomography and central venous pressure (CVP). Pericardiectomy was routinely performed by median sternotomy in all patients without the use of cardiopulmonary bypass. The extent of pericardiectomy included at least the anterolateral pericardium between the two phrenic nerves, the basal pericardium over the diaphragmatic surface, the pericardium on the great arteries and the pericardium from superior vena cava-right atrium junction to inferior vena cava-right atrium junction[14].

The primary outcome was the incidence of postoperative complications which were defined as the comorbidities that occurred after surgery but did not exist before. Second outcomes were postoperative intensive care unit (ICU) stay, postoperative hospital stay and in-hospital mortality. 


\section{Specimens analysis}

The expressions of MMP1, MMP2, MMP9 and TIMP1 mRNA were analyzed by quantitative real-time polymerase chain reaction (qRT-PCR) using Hieff UNICON® qPCR SYBR Green Master Mix (YISHEHG, Shanghai, China). GADPH mRNA was utilized as the endogenous control. Total RNA was isolated using Trizol reagent (Invitrogen) and complementary DNA was synthesized using Hifairß $\otimes 1$ st Strand cDNA Synthesis Kit (YISHEHG, Shanghai, China). Specific primers used for qRT-PCR assays were 5'GGGAGATCATCGGGACAACTC-3', 5'-GGGCCTGGTTGAAAAGCAT-3' for MMP1; 5'TGATCTTGACCAGAATACCATCGA-3', 5'- GGCTTGCGAGGGAAGAAGTT - 3' for MMP2; 5'GTGCTGGGCTGCTGCTTTGCTG-3', 5'-GTCGCCCTCAAAGGTTTGGAAT-3' for MMP9; 5'CTTCTGGCATCCTGTTGTTG-3', 5'-AGAAGGCCGTCTGTGGGT-3' for TIMP1; 5'TGCACCACCAACTGCTTAGC-3', 5'-GGCATGGACTGTGGTCATGAG-3' for GADPH.

\section{Statistical analysis}

The relative expressions of target genes were calculated by $\Delta \Delta C T$ method. The fold change in gene expression was calculated as $2^{-} \Delta \Delta C T$. The measurement data and the enumeration data were statistically analyzed with the Mann-Whitney $U$ test and the Fisher exact test, respectively. The receiver operating characteristic (ROC) curve and Youden Index were used to determine the cutoff values of MMPs or TIMPs predicting postoperative complications. Binary logistic regression analyses were performed to determine the correlation between MMPs/TIMPs and postoperative complications. Confounders were included, based on univariate analysis. These analyses were conducted using SPSS software (version 24.0, IBM SPSS Inc. United States). Statistical significance was set at $\mathrm{P}$ value $<0.05$ (all $\mathrm{P}$ values presented were two-sided).

\section{Results}

\section{Group division}

A total of 22 patients and corresponding pericardial specimens were enrolled in this study. A total of 12 postoperative complications were observed in 10 (45.5\%) patients (Supplemental Table 1), with one patient dying of cardiac failure on the fifth postoperative day. The most common complication was low cardiac output ( 7 cases), followed by cardiac failure ( 2 cases), acute kidney injury ( 2 cases) and pulmonary embolism ( 1 case). The patients were classified into two groups according to postoperative complications and we found that the patients with postoperative complications have higher level of MMP9 than those without postoperative complications $(P=0.002)$ (Table 1$)$. 
Table 1

Expressions of MMPs and TIMP1 in the constrictive pericardium based on the postoperative complications

\begin{tabular}{|llll|}
\hline Variables & \multicolumn{2}{l}{ Postoperative complications } & \multirow{2}{*}{ P value } \\
\cline { 2 - 3 } & Yes $(\mathrm{N}=10)$ & No $(\mathrm{N}=12)$ & \\
\hline MMP1 & $5.99(-0.44-11.79)$ & $9.16(2.96-13.06)$ & 0.539 \\
\hline MMP2 & $2.83(0.75-4.63)$ & $2.00(0.80-3.05)$ & 0.080 \\
\hline MMP9 & $5.77(3.19-11.11)$ & $3.32(2.04-4.85)$ & 0.002 \\
\hline TIMP1 & $1.53(-1.10-2.98)$ & $0.81(-0.54-2.55)$ & 0.180 \\
\hline Values presented as median (range). & & \\
\hline MMP, matrix metalloproteinase; TIMP, tissue inhibitors of matrix metalloproteinase \\
\hline
\end{tabular}

The result of ROC curve also presented the significant correlation between MMP9 and postoperative complications. The area under curve (AUC) was $0.883(95 \% \mathrm{Cl}=0.739-1.000, \mathrm{P}=0.002)$ (Fig. 1). The Youden Index was calculated and the result showed the optimal cutoff value of MMP9 level was 3.67 (sensitivity $90.0 \%$, specificity $83.3 \%$, Youden Index 0.733 ). According to the cutoff value, the patients were divided into the MMP9-low group (MMP9 <3.67) and the MMP9-high group (MMP9 $\geq 3.67$ ). There were no statistical differences between the two groups for gender, age, etiology, body mass index, cardiac functional class, preoperative CVP and other baseline characteristics (Table 2). 
Table 2

Baseline characteristics of study patients based on the level of MMP9 in the constrictive pericardium

\begin{tabular}{|c|c|c|c|}
\hline Variables & $\begin{array}{l}\text { MMP9-low group }(\mathrm{N}= \\
\text { 11) }\end{array}$ & $\begin{array}{l}\text { MMP9-high group }(\mathrm{N}= \\
\text { 11) }\end{array}$ & $\begin{array}{l}P \\
\text { value }\end{array}$ \\
\hline Gender & $11(100 \%)$ & $11(100 \%)$ & / \\
\hline \multicolumn{4}{|l|}{ Male } \\
\hline Age, years & $70(54-80)$ & $73(54-80)$ & 0.270 \\
\hline Etiology & & & / \\
\hline Tuberculosis & $11(100 \%)$ & $11(100 \%)$ & \\
\hline \multicolumn{2}{|l|}{$\begin{array}{l}\text { Preoperative NYHA functional } \\
\text { class }\end{array}$} & & 0.327 \\
\hline ૧ & $2(18.2 \%)$ & $0(0 \%)$ & \\
\hline ૧ & $1(9.1 \%)$ & $3(27.3 \%)$ & \\
\hline प & $8(72.7 \%)$ & $8(72.7 \%)$ & \\
\hline Hypertension & $1(9.1 \%)$ & $2(18.2 \%)$ & 1.000 \\
\hline Diabetes & $2(18.2 \%)$ & $2(18.2 \%)$ & 1.000 \\
\hline Heart disease* & $2(18.2 \%)$ & $4(36.4 \%)$ & 0.635 \\
\hline $\mathrm{BMI}, \mathrm{kg} / \mathrm{m}^{2}$ & $21.3(17.3-24.8)$ & $21.0(17.7-24.8)$ & 0.652 \\
\hline Pulse rate, beats/min & $98(80-112)$ & $90(80-145)$ & 0.699 \\
\hline Preoperative $\mathrm{CVP}, \mathrm{cmH}_{2} \mathrm{O}$ & $23.0(20.5-30.0)$ & $30.0(20.4-42.5)$ & 0.065 \\
\hline Pericardial thickness, mm & $10.7(8.2-16.0)$ & $10.7(7.0-16.0)$ & 0.847 \\
\hline LVEF, \% & $54.3(50.0-56.4)$ & $56.0(51.9-64.0)$ & 0.101 \\
\hline CRP, mg/L & $11.7(5.0-21.0)$ & $17.0(5.0-62.5)$ & 0.076 \\
\hline $\mathrm{ESR}, \mathrm{mm} / \mathrm{h}$ & $37.0(3.0-52.0)$ & $37.0(17.0-68.0)$ & 0.300 \\
\hline \multicolumn{4}{|c|}{$\begin{array}{l}\text { Values presented as } \mathrm{N} \text { (percentage) for categorical variables and median (range) for continuous } \\
\text { variables. }\end{array}$} \\
\hline \multicolumn{4}{|c|}{ MMP9-low was defined as the level of MMP9 $<3.67$ and MMP9-high was defined as MMP9 $\geq 3.67$. } \\
\hline \multicolumn{4}{|c|}{$\begin{array}{l}\text { MMP, matrix metalloproteinase; NYHA, New York Heart Association; BMI, body mass index; CVP, } \\
\text { central venous pressure; LVEF, left ventricular ejection fraction (measured on echocardiogram); CRP, C- } \\
\text { reactive protein; ESR, erythrocyte sedimentation rate; }\end{array}$} \\
\hline
\end{tabular}


The comparison of outcomes between the MMP9-low group and the MMP9-high group was shown in Table 3. Compared with the MMP9-high group, the MMP9-low group had significantly lower incidence of postoperative complications ( $9.1 \%$ vs. $81.8 \%, P=0.002)$. In addition, the MMP9-low group had shorter postoperative ICU stay $(P=0.040)$ and postoperative hospital stay $(P=0.043)$ in comparison to the MMP9-high group. One in-hospital death occurred in the MMP9-high group with no mortality in the MMP9-low group.

Table 3

Postoperative outcomes of study patients stratified by the level of MMP9 in the constrictive pericardium

\begin{tabular}{|c|c|c|c|}
\hline Variables & $\begin{array}{l}\text { MMP9-low group }(\mathrm{N}= \\
\text { 11) }\end{array}$ & $\begin{array}{l}\text { MMP9-high group }(\mathrm{N}= \\
\text { 11) }\end{array}$ & $\begin{array}{l}P \\
\text { value }\end{array}$ \\
\hline Postoperative $\mathrm{CVP}, \mathrm{cmH}_{2} \mathrm{O}$ & $17.0(5.0-20.0)$ & $17.8(5.0-32.0)$ & 0.365 \\
\hline Postoperative intubation, $\mathrm{h}$ & $11(3-132)$ & $28(5-232)$ & 0.173 \\
\hline $\begin{array}{l}\text { Duration of using vasoactive } \\
\text { agents, } h\end{array}$ & $0(0-116)$ & $47(0-143)$ & 0.114 \\
\hline Postoperative complications & $1(9.1 \%)$ & $9(81.8 \%)$ & 0.002 \\
\hline Postoperative ICU stay, days & $2(1-5)$ & $4.5(1-10)$ & 0.040 \\
\hline Postoperative hospital stay, days & $18(13-24)$ & $23(13-29)$ & 0.043 \\
\hline In-hospital mortality & $0(0 \%)$ & $1(9.1 \%)$ & 1.000 \\
\hline \multicolumn{4}{|c|}{$\begin{array}{l}\text { Values presented as median (range) for continuous variables and } \mathrm{N} \text { (percentage) for categorical } \\
\text { variables. }\end{array}$} \\
\hline \multicolumn{4}{|c|}{ MMP9-low was defined as the level of MMP9 $<3.67$ and MMP9-high was defined as MMP9 $\geq 3.67$. } \\
\hline \multicolumn{4}{|c|}{ MMP, matrix metalloproteinase; CVP, central venous pressure; ICU, intensive care unit } \\
\hline
\end{tabular}

\section{Multivariate analysis}

In order to determine the degree of contribution of MMP9 on postoperative outcomes, the statistically significant factors in univariate analysis were included in multivariate regression model (Supplemental Table 2). Binary logistic regression analysis demonstrated that compared with the MMP9-low group, the risk for postoperative complications significantly increased in the MMP9-high group (OR, 27.096, 95\% Cl 1.166-629.886, $\mathrm{P}=0.040$ ) (Table 4). 
Table 4

Effect of the level of MMP9 in the constrictive pericardium on postoperative outcomes

\begin{tabular}{|llll|}
\hline Groups & \multicolumn{3}{l|}{ Postoperative complications } \\
\cline { 2 - 4 } & OR & $95 \% \mathrm{Cl}$ & P value \\
\hline MMP9-low group & 1 & $/$ & $/$ \\
\hline MMP9-high group & 27.096 & $1.166-629.886$ & 0.040 \\
\hline MMP9-low was defined as the level of MMP9 < 3.67 and MMP9-high was defined as MMP9 $\geq 3.67$. \\
\hline MMP, matrix metalloproteinase; OR, odds ratio; Cl, confidence interval \\
\hline
\end{tabular}

\section{Discussion}

Surgical pericardiectomy is the curative treatment for constrictive pericarditis and is strongly suggested in the patients with progressive symptoms after medical therapy[15]. Despite being considered effective, pericardiectomy is associated with non-negligible risk of postoperative complications and in-hospital mortality. There have been a number of studies exploring the risk factors of poor outcomes after pericardiectomy, and the results showed that surgical outcomes depended heavily on the functional status of patients, the etiology of constrictive pericarditis, the timing of surgical intervention, the extent of pericardial resection and the need for cardiopulmonary bypass[16-20]. However, most of the studies represented single-center and retrospective experiences. Tzani, A. et al. performed a meta-analysis to systematically review the clinical outcomes of patients undergoing pericardiectomy for constrictive pericarditis[21]. This meta-analysis included 27 eligible studies and 2,114 patients. The results highlighted that radiation and after-cardiac surgery patients had a significantly high mortality risk, and that surgical intervention should be considered before advanced symptoms occurred, and that total pericardiectomy without the use cardiopulmonary bypass was preferred.

However, the association between abnormal gene expressions in pericardium and postoperative outcomes has not been investigated. MMPs are well-known mediators of cardiovascular pathophysiology. The changes of MMPs expressions are directly associated with inflammation and the subsequent formation of fibrosis, a key pathological process of many diseases including constrictive pericarditis[22, 23]. This study first evaluated the associations of MMPs expressions in pericardial tissues with postoperative outcomes in patients undergoing pericardiectomy for constrictive pericarditis. We found a positive correlation between the level of MMP9 and postoperative outcomes. The results showed that overexpression of MMP9 could increase the incidence of postoperative complications and prolong the length of postoperative ICU and hospital stay. Multivariate analysis further verified that high level of MMP9 in the pericardium was the independent risk factor of postoperative complications. It was worth mentioning that the predictive value for postoperative complications was significant when the cutoff value of MMP9 level was 3.67 , with $90.0 \%$ sensitivity and $83.3 \%$ specificity. 
The major postoperative complication in this study was low cardiac output which mainly resulted from the presence of myocardial fibrosis and atrophy. Previous studies indicated that MMP9 played an important role in tissue remodeling related to cardiac function because of its central role in inflammation and elastin degradation which led to decreased elasticity[23, 24]. In addition, enhanced activation of MMP9 was associated with the state of active myocardial remodeling and could be a potentially useful marker for the identification of patients at risk for heart failure development and poor outcome[25].

There are some limitations that should be pointed out. Firstly, as this is a single-center retrospective study, the selection bias is inevitable. Secondly, the sample size is small, which may interfere in our findings. It is difficult to increase sample size in a small period of time due to the low incidence of constrictive pericarditis. Finally, lacking of normal tissue analysis may also influence our findings.

\section{Conclusions}

Our study has shown that although effective at relieving symptoms of constrictive pericarditis, pericardiectomy was associated with high incidence of postoperative complications. Increased expression of MMP9 in the pericardium was significantly associated with poor postoperative outcomes and was the independent risk factor of postoperative complications. The level of MMP9 could be used as a potential marker for prediction of surgical outcomes.

\section{List Of Abbreviations}

MMPs, matrix metalloproteases; TIMPs, tissue inhibitors of metalloproteinases; CVP, central venous pressure; ICU, intensive care unit; qRT-PCR, quantitative real-time polymerase chain reaction; ROC, receiver operating characteristic; AUC, area under curve

\section{Declarations}

\section{Ethics approval and consent to participate}

The study protocol was approved by the Institutional Review Board of Affiliated Hangzhou Chest Hospital, Zhejiang University School of Medicine (ID of ethics approval: 20160830) and written patient informed consent was obtained.

\section{Consent for publication}

Not applicable.

\section{Availability of data and materials}

The datasets used during the current study are available from the corresponding author on reasonable request. 


\section{Competing interests}

All authors declare that they have no competing interests.

\section{Funding}

No funding.

\section{Author Contributions}

Drs. LF and GC contributed to the conception and design of the work. Drs. LF and GY contributed to data analysis and manuscript editing. Drs. WY and BY contributed to data acquisition, statistical analysis and interpretation of the data. Drs. LF and GC contributed to the revision of the manuscript. All authors have approved the final draft of the manuscript.

\section{Acknowledgments}

Not applicable

\section{References}

1. Welch TD, Oh JK. Constrictive Pericarditis. Cardiology clinics. 2017;35(4):539-49.

2. Welch TD. Constrictive pericarditis: diagnosis, management and clinical outcomes. Heart (British Cardiac Society). 2018;104(9):725-31.

3. Tuck BC, Townsley MM. Clinical Update in Pericardial Diseases. Journal of cardiothoracic and vascular anesthesia. 2019;33(1):184-99.

4. Miranda WR, Oh JK. Constrictive Pericarditis: A Practical Clinical Approach. Progress in cardiovascular diseases. 2017;59(4):369-79.

5. Khandaker $\mathrm{MH}$, Schaff HV, Greason $\mathrm{KL}$, et al. Pericardiectomy vs medical management in patients with relapsing pericarditis. Mayo Clin Proc. 2012;87(11):1062-70.

6. Syed FF, Schaff HV, Oh JK. Constrictive pericarditis-a curable diastolic heart failure. Nature reviews Cardiology. 2014;11(9):530-44.

7. Gopaldas RR, Dao TK, Caron NR, et al. Predictors of in-hospital complications after pericardiectomy: a nationwide outcomes study. J Thorac Cardiovasc Surg. 2013;145(5):1227-33.

8. Liu VC, Fritz AV, Burtoft MA, et al. Pericardiectomy for Constrictive Pericarditis: Analysis of Outcomes. Journal of cardiothoracic and vascular anesthesia. 2021;S1053-0770(21):00115-4.

9. Visse R, Nagase H. Matrix metalloproteinases and tissue inhibitors of metalloproteinases: structure, function, and biochemistry. Circulation research. 2003;92(8):827-39.

10. Amalinei C, Caruntu ID, Giusca SE, et al. Matrix metalloproteinases involvement in pathologic conditions. Romanian journal of morphology and embryology = Revue roumaine de morphologie et embryologie. 2010;51(2):215-28. 
11. Reis ST, Leite KRM, Viana NI, et al. MMP9 overexpression is associated with good surgical outcome in children with UPJO: Preliminary results. BMC Urol. 2016;16(1):44.

12. Garcia-Prieto CF, Gil-Ortega M, Vega-Martin E, et al. Beneficial Effect of Bariatric Surgery on Abnormal MMP-9 and AMPK Activities: Potential Markers of Obesity-Related CV Risk. Front Physiol. 2019;10:553.

13. Moreno-Ajona D, Irimia P, Rodriguez JA, et al. Elevated circulating metalloproteinase 7 predicts recurrent cardiovascular events in patients with carotid stenosis: a prospective cohort study. BMC Cardiovasc Disord. 2020;20(1):93.

14. Huang J, Zhu P, Zhong F, et al. Clinical significance of pulse index contour continuous cardiac output monitoring in patients with constrictive pericarditis undergoing pericardiectomy. Interactive cardiovascular and thoracic surgery. 2020;31(3):364-8.

15. Depboylu BC, Mootoosamy P, Vistarini N, et al. Surgical Treatment of Constrictive Pericarditis. Texas Heart Institute journal. 2017;44(2):101-6.

16. Fang LK, Yu GC, Huang JP, et al. Predictors of postoperative complication and prolonged intensive care unit stay after complete pericardiectomy in tuberculous constrictive pericarditis. Journal of Cardiothoracic Surgery. 2020;15(1).

17. Nishimura S, Izumi C, Amano M, et al. Long-Term Clinical Outcomes and Prognostic Factors After Pericardiectomy for Constrictive Pericarditis in a Japanese Population. Circulation journal: official journal of the Japanese Circulation Society. 2017;81(2):206-12.

18. Murashita T, Schaff HV, Daly RC, et al. Experience With Pericardiectomy for Constrictive Pericarditis Over Eight Decades. The Annals of thoracic surgery. 2017;104(3):742-50.

19. Choi MS, Jeong DS, Oh JK, et al. Long-term results of radical pericardiectomy for constrictive pericarditis in Korean population. J Cardiothorac Surg. 2019;14(1):32.

20. Rupprecht L, Putz C, Florchinger B, et al. Pericardiectomy for Constrictive Pericarditis: An Institution's 21 Years Experience. The Thoracic and cardiovascular surgeon. 2018;66(8):645-50.

21. Tzani A, Doulamis IP, Tzoumas A, et al. Meta-Analysis of Population Characteristics and Outcomes of Patients Undergoing Pericardiectomy for Constrictive Pericarditis. The American journal of cardiology. 2021.

22. Merkle M, Ribeiro A, Sauter M, et al. Effect of activation of viral receptors on the gelatinases MMP-2 and MMP-9 in human mesothelial cells. Matrix Biol. 2010;29(3):202-8.

23. Cabral-Pacheco GA, Garza-Veloz I, Castruita-De la Rosa C, et al. The Roles of Matrix Metalloproteinases and Their Inhibitors in Human Diseases. International journal of molecular sciences. 2020;21(24).

24. Lindsey ML. Assigning matrix metalloproteinase roles in ischaemic cardiac remodelling. Nature reviews Cardiology. 2018;15(8):471-9.

25. Radosinska J, Barancik M, Vrbjar N. Heart failure and role of circulating MMP-2 and MMP-9. Panminerva Med. 2017;59(3):241-53. 
Figures

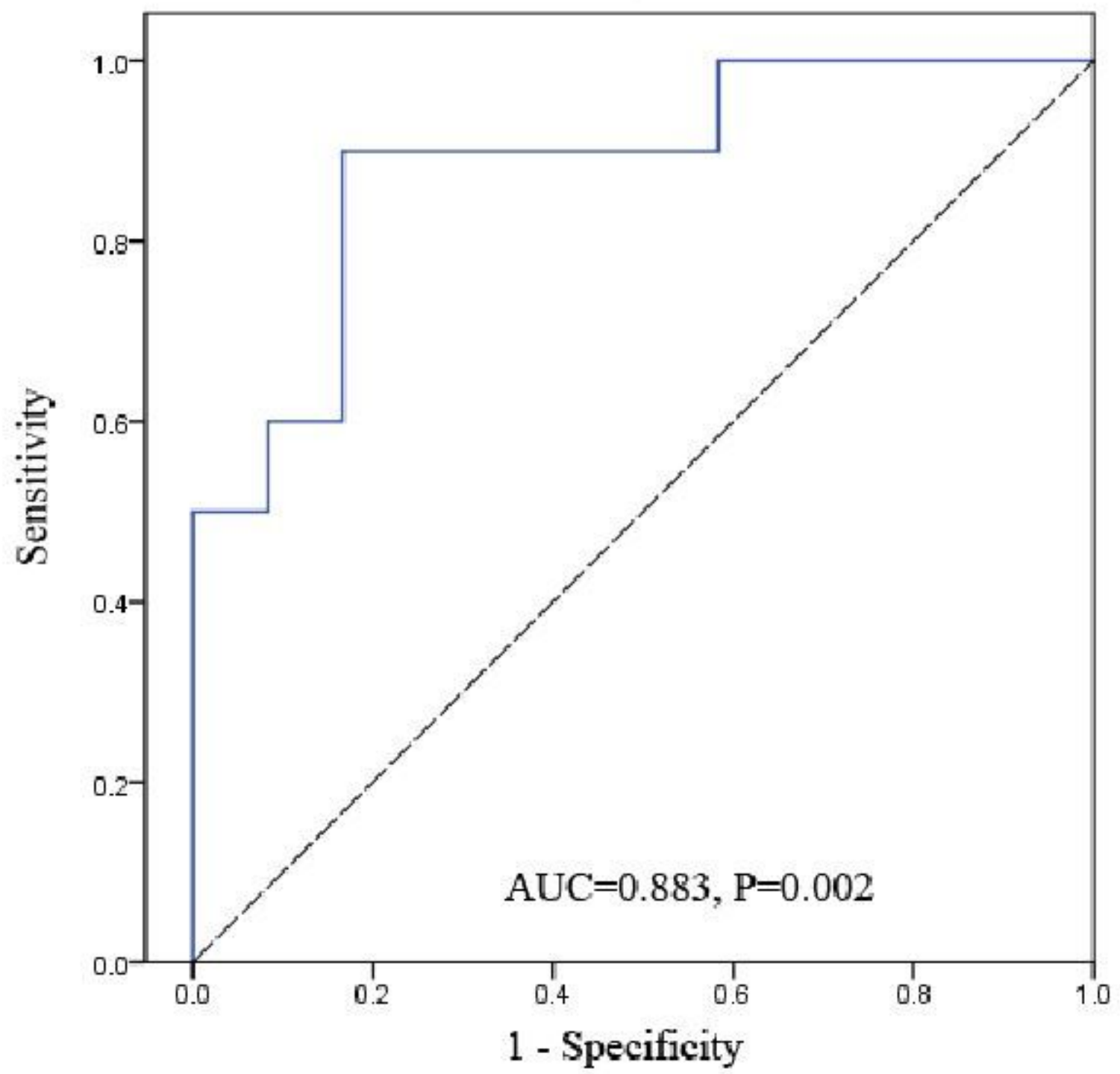

Figure 1

The area under the ROC curve for postoperative complications determined using the level of MMP9. ROC, receiver operating characteristic; AUC, area under the curve.

\section{Supplementary Files}

This is a list of supplementary files associated with this preprint. Click to download.

- Supplementaltable.docx 